FORMATION Formation emploi

Revue française de sciences sociales

114 | Avril-juin 2011

Pêle-mêle

\title{
Développer des compétences ou comment s'engager dans l'agir professionnel
}

Skills development or how to engage in professional action?

Entwicklung von Kompetenzen

oder wie geschieht der Übergang zum beruflichen Handeln?

Desarrollar competencias o cómo emprender la acción profesional

\section{Najoua Mohib}

\section{OpenEdition}

Journals

Édition électronique

URL : https://journals.openedition.org/formationemploi/3378

DOI : 10.4000/formationemploi.3378

ISSN : 2107-0946

Éditeur

La Documentation française

Édition imprimée

Date de publication : 31 juillet 2011

Pagination : 55-71

ISSN : 0759-6340

Référence électronique

Najoua Mohib, « Développer des compétences ou comment s'engager dans l'agir professionnel », Formation emploi [En ligne], 114 | Avril-juin 2011, mis en ligne le 04 octobre 2011, consulté le 28 juin 2022. URL : http://journals.openedition.org/formationemploi/3378; DOI : https://doi.org/10.4000/ formationemploi.3378

(c) Tous droits réservés 


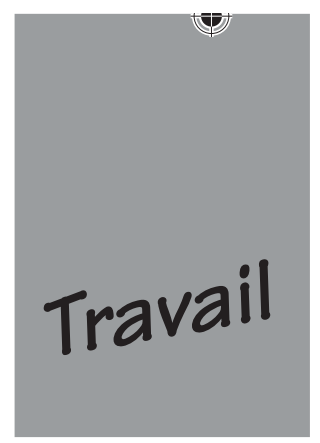

\title{
Développer des compétences ou comment s'engager dans l'agir professionnel
}

Najoua Mohib*

\begin{abstract}
Le développement des compétences professionnelles est ici abordé sous l'angle du pouvoir d'agir, en confrontant des modèles de formation issus de champs professionnels différents : enseignants, ingénieurs, compagnons.
\end{abstract}

L'histoire du travail montre que le système traditionnel de production s'est modifié depuis une trentaine d'années. Face à la nécessité de s'adapter aux profondes mutations socio-économiques, les entreprises privilégient la mise en place d'organisations de travail favorisant le renouvellement des compétences de leurs salariés. L'introduction des TIC (Technologies de l'information et de la communication), la modification du statut du facteur humain ou encore la pression de l'efficacité exigent de la formation qu'elle soit aussi professionnalisante que possible. Dans ce contexte, la question des compétences, comme ressources à développer et dont la valeur marchande ne peut plus être niée, occupe une place centrale dans la conception des espaces visant la transformation des « sujets humains » (Barbier, 2000).

On peut dater aux années 90 l'émergence d'un nouveau modèle dans le champ de la formation professionnelle, qui concrétise la mise en place de pratiques de formation orientées vers la compétitivité et l'emploi. Fortement controversée en raison de sa dérive instrumentale, la logique compétence a néanmoins contribué à faire évoluer l'appareil de formation. Sans aller jusqu'à considérer ce modèle comme «révolutionnaire 》 voire «novateur» (Livian \& Terrenoire, 1995), des auteurs comme

* Najoua Mohib est maître de conférences en sciences de l'éducation à l'université de Strasbourg, chercheur au LISEC (Laboratoire interuniversitaire des sciences de l'éducation et de la communication - EA2310). Ses travaux portent sur les pratiques de formation initiale et professionnelle et se focalisent notamment sur l'accompagnement et le développement des compétences. Plus récemment, ses recherches interrogent les liens entre compétences et technologies éducatives. Ses dernières publications : Mohib N. (Éd.) (2010), TIC et développement des compétences : quelles réciprocités ? Questions Vives, volume 7, numéro 14, Aix en Provence, Éditions En Question. Mohib N., Sonntag M., Werckmann F. (2010), "Le tutorat méthodologique en école d'ingénieurs: pourquoi est-ce encore une bonne idée? ", Actes d'AREF 2010, Genève. 
Dubar (1996), Dugué (1999), ou encore Paradeise et Lichtenberger (2001) reconnaissent qu'il implique la mise en œuvre de nouvelles pratiques de formation. De ce point de vue, cette approche a ouvert le champ à une série de démarches construites autour de l'autonomisation du sujet s'agissant du développement de ses potentialités. Symbolisée par le bilan de compétence, ou plus récemment la VAE (Validation des acquis de l'expérience), la logique compétence conduit à inscrire l'individu dans une perspective de formation permanente, à son initiative et sous sa responsabilité. En même temps qu'ils contribuent à redéfinir la place du sujet dans la société, les dispositifs de formation revendiquent un rapprochement avec le travail et reconnaissent «l'importance de l'agir comme occasion d'apprendre » (Astier, 2009).

Sur le plan de la recherche, deux types de travaux portant sur la problématique du lien entre formation et compétence peuvent être identifiés. Les premiers, menés essentiellement dans le champ de la sociologie et de la gestion, portent sur l'analyse de la logique compétence qui permet de penser le nouveau modèle d'organisation apprenante (Dietrich, 1999 ; Dugué, 1994 ; Lichtenberger, 1999 ; Oiry \& Iribarne (d'), 2001). Une deuxième catégorie renvoie à des recherches de type éducationiste qui tentent de comprendre comment s'acquièrent et se développent les compétences (professionnelles, relationnelles, sociales, etc.) (Olry, 2003 ; Pastré, 1999 ; Wittorski, 1998). Notre recherche sur les actions de développement des compétences professionnelles au sein de dispositifs visant à professionnaliser des acteurs s'inscrit dans cette seconde optique.

Par rapport à la plupart des travaux qui cherchent principalement à identifier les savoirs mobilisés et combinés dans l'action efficace (Rabardel \& Six, 1995 ; Perrenoud, 2004), notre étude explore l'engagement même de l'individu dans l'action. Certes, l'organisation d'activités de formation favorisant l'acquisition des compétences professionnelles suppose «qu'on [les] ait identifiées et qu'on sache comment elles se construisent » (Perrenoud et al., 1998). Cependant, dans la mesure où la compétence implique l'engagement d'un acteur dans une action reconnue efficace, il importe d'examiner plus avant les processus à l'œuvre dans le passage de la prise de décision vers la réalisation effective.
Si la compétence est de toute évidence indissociable de l'action, elle "émerge d'un effort d'adaptation aux contraintes d'une situation dans laquelle l'individu puise des ressources pour agir » (Durand et al., 2002). Ainsi, il ne suffit pas de savoir ou de vouloir agir, il faut également pouvoir agir, non pas au sens d'être autorisé à faire mais de s'autoriser soi-même à agir. C'est à cette dernière dimension, encore peu explorée ${ }^{1}$, que s'attache notre étude. Plutôt que de nous centrer sur les facteurs cognitifs et motivationnels, l'exploration de ce qui permet à l'individu de s'engager dans l'action nous paraît constituer une piste particulièrement intéressante pour comprendre comment se construisent les compétences. Il s'agit, dans le cadre de la présente contribution, de mettre au jour ce qui favorise l'engagement dans l'agir professionnel, tant du point de vue des acteurs qui conçoivent et organisent les activités de formation, que du point de vue des acteurs engagés eux-mêmes dans cette dynamique. Pour ce faire, nous avons retenu trois espaces de formation afférents au système éducatif au sein desquels la question du développement des compétences professionnelles constitue une préoccupation centrale : les IUFM (Instituts universitaires de formation des maîtres), les écoles d'ingénieurs et la maison des compagnons du devoir.

Dans un premier temps, nous présenterons les logiques qui sous-tendent les activités de formation. Nous définirons également ici la notion de " compétence professionnelle », que nous aborderons sous l'angle de «l'engagement dans l'agir ». Dans un deuxième temps, nous indiquerons le choix des terrains d'investigation et les modalités de collecte et d'analyse des données. Dans un troisième temps, nous chercherons à identifier les conditions qui favorisent l'engagement dans l'agir professionnel, telles qu'elles sont prévues et perçues par l'institution mais aussi telles qu'elles sont vécues par les anciens stagiaires, avant de confronter l'ensemble des discours dans la dernière partie de ce texte. Les résultats permettront de discuter la place de l'Autre, et plus particulièrement celle de l'accompagnateur, à l'ère du nouveau paradigme

\footnotetext{
${ }^{1}$ Dans une toute autre approche, le «pouvoir d'agir» fait l'objet d'un intérêt particulier dans les champs de la clinique de l'activité et de la didactique professionnelle. $C f$. Clot Y. (2008). Travail et pouvoir d'agir, Paris, PUF ou encore Pastré P., Rabardel P. (2005), Modèles du sujet pour la conception. Dialectiques activités développement, Toulouse, Octarès.
} 
social de «l'individu acteur et auteur de sa propre vie » (Wittorski, 2009).

\section{DÉVELOPPEMENT DES COMPÉTENCES PROFESSIONNELLES : UNE NOUVELLE CONCEPTION DE LA FORMATION}

Il est communément admis que les compétences professionnelles se développent aussi bien en dehors que dans le cadre de la formation. Notre travail de recherche se rapporte précisément au champ de la formation. Après une brève évocation du contexte d'émergence des «cultures du développement des compétences » (Barbier, 2009), nous présenterons les grandes logiques qui traversent l'ensemble des espaces de formation (professionnel, social, éducatif). Sans chercher à rendre compte de la diversité des dispositifs de développement des compétences professionnelles, nous indiquerons trois orientations principales à partir d'une analyse des différentes formes d'activités de formation. Ces éléments nous permettront d'aborder, à la fin de cette partie, la problématique du développement des compétences professionnelles sous l'angle de l'engagement dans l'agir.

\section{Pour une « théorie de l'acteur»}

Depuis l'avènement de la loi du 4 mai 2004, les programmes de formation professionnelle sont désormais axés sur le «développement des compétences » afin de « contribuer au développement culturel, économique et à la promotion sociale $»^{2}$. Plus concrètement, les programmes d'études professionnelles (initiales et continues) sont passés d'une conception calquée sur le système scolaire à une conception fondée sur une approche plus flexible, articulant transmission des savoirs et construction des compétences. Dans ce cadre, les espaces de formation promeuvent l'idée d'un apprenant devenu acteur de son savoir, de son projet professionnel et de sa formation. Autrement dit,

${ }^{2}$ Loi $n^{\circ} 2004-391$ du 4 mai 2004 relative à la formation professionnelle tout au long de la vie et au dialogue social, publiée au JO du 5 mai 2004. le développement des compétences professionnelles serait le fait des sujets eux-mêmes qui s'emparent des potentialités humaines ou matérielles de la situation, au gré d'une réflexion (individuelle et collective) sur l'expérience. Ces démarches ne sont pas complètement nouvelles. Elles s'inscrivent dans un renouvellement des théories sur lesquelles reposent les multiples formes de formation.

Cependant, cette conception, qui s'inspire dans une large mesure des apports théoriques développés par Piaget et Vygotski, est à l'origine d'une nouvelle culture pédagogique dans le milieu de la formation professionnalisante. Plusieurs auteurs parlent ainsi d'un «nouveau paradigme de la formation $»^{3}$ dont «l'un des signes les plus nets serait l'effacement progressif de la logique enseignement sous la poussée d'une logique apprentissage » (Carré, 2005). Le paradigme de la « construction $»^{4}$, qui domine aujourd'hui dans le champ de la formation, n'exclut pas la forme transmissive du modèle pédagogique. Il reconsidère simplement la position hégémonique de celle-ci et considère l'acte d'appendre comme une condition à la fois supérieure à l'acte d'enseigner et nécessaire au développement des compétences professionnelles du sujet : «Le savoir ou le savoir-faire ne se déverse pas. Vous ne retenez pas ce que l'on vous a enseigné, mais ce que vous avez appris. " (Albertini cité dans Carré, 2005)

Dans ce nouveau paradigme, où le vocabulaire habituellement employé pour décrire les situations pédagogiques s'est lui-même transformé (Astolfi, 2003), il apparaît que les mots « compétence », " professionnel», et «apprentissage » sont devenus les concepts clés qui sous-tendent les systèmes actuels de formation. Les formations professionnelles telles qu'elles sont élaborées, ces dernières années, renvoient à des dispositifs discontinus où le rapport au savoir

\footnotetext{
3 Par exemple, (Baillauquès, 2001); (Carré, 2005); (Raucent \& Vander Borght, 2006).

${ }^{4}$ Dans la plupart des pays occidentaux, les systèmes de formation (initiale et continue) sont passés d'un modèle centré sur la transmission des savoirs, où le rapport au savoir semble totalement médiatisé par le «maître » (Astolfi, 2003), à un modèle centré sur la construction des connaissances et le développement des compétences qui reconnaît la position active de l'apprenant. Aujourd'hui, les programmes d'études professionnelles reposent sur l'idée que c'est l'apprentissage et non plus la transmission (envisagée désormais comme un acte de co-construction) qui crée les conditions nécessaires au développement cognitif des individus.
} 
passe d'abord par l'apprenant, perçu comme l'acteur principal de sa formation, capable de saisir des opportunités d'apprentissage pour se construire. Considéré jadis comme un «modèle à imiter », l'accompagnateur (tuteur, conseiller, superviseur, facilitateur, guide, médiateur ou plus généralement une personne qui fait autorité) est devenu progressivement un « appreneur » actif dans la construction de la professionnalité des apprenants. C'est dans ce contexte que se développent aujourd'hui des nouvelles formes de formation articulant situations de travail et situations de formation en vue de favoriser la construction individuelle des compétences professionnelles.

\section{Trois façons d'envisager le développement des compétences professionnelles}

Le recours aux situations de travail dans les pratiques de formation n'est pas récent; pourtant, c'est sur cette conception que se fondent actuellement les programmes novateurs des dispositifs de développement des compétences professionnelles. Pourquoi parle-t-on de nouvelles formes de formation alors que celles-ci ont toujours été centrées sur l'idée d'une adaptation à l'évolution des situations professionnelles ? Pour Barbier (2001), «le phénomène nouveau est ici ce que nous pourrions appeler le développement d'une pression à accroître la cohérence entre formation et environnement d'action ». Autrement dit, qu'il s'agisse de démarches mises en œuvre par des organisations, des entreprises ou encore des établissements d'enseignement scolaire et supérieur, toutes tentent de prendre en compte la réalité des contextes de travail. Trois façons d'envisager le développement des compétences professionnelles semblent ainsi émerger : des activités de formation qui s'élaborent à partir des situations de travail (par ex. : analyse du travail, échanges de pratiques, recherche-formation, etc.), dans des situations de travail (par ex. : coaching, mentoring, communauté de pratique, etc.) et enfin par des mises en situation de travail (par ex. : simulation, stage, etc.).

\section{Former à partir des situations de travail}

Dans ce type de formation conçu à partir des pratiques réelles, l'individu est conduit non seulement à explorer les lois de son action, c'est-à-dire à identifier les règles qui gouvernent ses actions, mais aussi à développer des stratégies pour faire face à des situations nouvelles. Cette conception repose sur l'idée que l'activité constitue à la fois un objet d'investigation et un outil de développement au service de la formation. Le fait de critiquer, d'objectiver, d'interpréter son propre vécu professionnel transformerait le savoir agir en pouvoir d'agir. Au fond, la réflexivité générée à partir d'une mise en représentation (mentalisation) et d'une mise en discours (formalisation) de l'activité (Barbier, 2009) favoriserait la construction des compétences professionnelles. Parmi les approches actuelles qui se développent dans les différents champs de la formation, on pourra citer, entre autres, « l'analyse du travail $»^{5}$ ou encore «l'analyse des pratiques professionnelles $»^{6}$.

\section{Former dans les situations de travail}

La deuxième grande orientation concerne plutôt le champ des organisations. Il s'agit plus précisément d'actions de formation intégrées aux activités de production. Elles s'adressent à des salariés ou des collectifs de travail réunis au sein de groupes créés spécifiquement à cette occasion. L'objectif premier est l'optimisation de la formation des individus afin d'améliorer l'efficacité et la performance de l'entreprise ou l'organisation. Les groupes de progrès, le coaching, l'action learning ou encore les cercles de $q_{\text {qualité }}^{7}$ représentent quelques-uns de ces dispositifs qui exploitent la capacité formative des situations de travail. Toutefois, au-delà de leur intention performative, ces démarches intégrées au travail présentent également des effets potentiellement formatifs pour

\footnotetext{
${ }^{5}$ Appliquée dans différents domaines professionnels, l'analyse du travail repose sur une méthode de recherche développée en psychologie et ergonomie. Elle est notamment utilisée comme un moyen pour placer l'individu en posture d'analyse dans la perspective d'une transformation de son rapport à l'activité. Pour en savoir plus sur cette approche, $c f$. (Durand, 2009).

${ }^{6}$ De manière générale, l'analyse des pratiques professionnelles désigne à la fois une technique et un objectif. Elle vise, à travers différentes modalités, la production des savoirs sur l'action (fonction théorique) et la professionnalisation des acteurs (fonction opératoire). Cette démarche polyfonctionnelle est particulièrement bien synthétisée dans l'article de Marcel et al. (2002).

7 Fondés sur des démarches différentes, tous ces dispositifs reposent sur la mise en place de situations réflexives individuelles et/ou collectives visant à l'élaboration d'un nouveau cadre de perception de l'activité afin d'élargir les capacités d'action de chaque individu.
} 
les individus dans la mesure où elles constituent « un des moyens du processus d'auto-transformation des compétences qu'[ils mettent] en auvre » (Barbier et al., 1996).

\section{Former par une mise en situation de travail}

Enfin, une troisième manière de former aux compétences professionnelles consiste à concevoir les activités de formation à partir d'une mise en situation de travail. Une telle configuration repose sur l'idée assez simple que l'on peut se préparer à une activité professionnelle en étant au contact de cette activité ou de son environnement. Ce n'est ni sur les bancs de l'université que le futur médecin apprendra à soigner des malades ni en se limitant à des séminaires consacrés aux théories de l'apprentissage que le futur enseignant parviendra à gérer sa classe. Concrètement, cette mise en situation de travail trouve son expression dans plusieurs formules (simulation, conception et conduite de projet, etc.) dont la plus emblématique est celle du stage, utilisée initialement dans la formation des apprentis et la formation continue des adultes (Pelpel, 2001). Sans faire l'inventaire des différents dispositifs, on retrouve là aussi bien les nouvelles formes d' « immersion » (Barbier et al., 1996) mises en place dans les organisations de travail que les stages de spécialisation, par exemple, introduits plus récemment dans le cadre des formations professionnelles universitaires.

Ainsi, les dispositifs de formation permettent de développer une véritable « pensée pour l'action », à travers les différents types de démarches mises en place pour prendre en charge le développement des compétences professionnelles. Plus qu'un simple rapprochement, il faut voir ici une véritable articulation de la relation travail-formation, favorisée par les activités conçues à partir, dans et par la pratique.

Aujourd'hui, la formation comme espace intentionnel de professionnalisation des individus tente de développer l'autonomie des apprenants. Ainsi, les principales modalités formatives que nous avons mises en évidence plus haut reposent sur l'injonction à la réflexivité. Certes, apprendre à observer, à analyser ou à réfléchir sur l'agir professionnel permet de poser des actes efficaces, d'améliorer sa pratique, d'augmenter son professionnalisme. Toutefois, la mise en œuvre des connaissances préalables ne suffit pas pour développer des compétences professionnelles. Il ne suffit pas de savoir comment agir, ni même de vouloir agir, il faut également pouvoir agir. Aussi, quels sont les processus qui soutiennent l'engagement de l'individu dans l'agir professionnel orienté vers l'efficacité et la reconnaissance de l'acte posé ? Il convient de préciser les termes de notre problématique avant de présenter le cadre de notre démarche empirique.

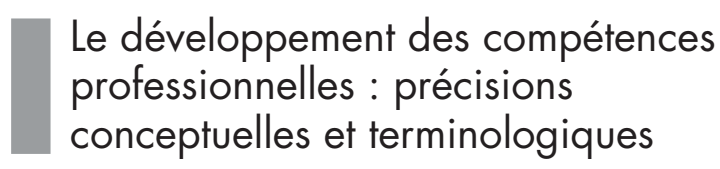

Le développement des compétences professionnelles suscite, aujourd'hui, au même titre que la question de leur évaluation et de leur reconnaissance, un intérêt majeur pour la recherche menée dans le champ de la formation des adultes. Pour résumer, il existe deux manières d'aborder la question du développement des compétences professionnelles. La première consiste à se demander comment se forment les compétences professionnelles (approche identitaire) ; dans la seconde, il s'agit plutôt de savoir comment former aux compétences professionnelles (approche instrumentale). Bien sûr, les deux approches ne sont pas indépendantes; l'intérêt de notre étude réside sans doute dans le lien qu'elle tente d'établir entre l'analyse, d'une part, des modalités de formation qui organisent les actions de développement des compétences professionnelles et, d'autre part, des processus qui sous-tendent cette dynamique de construction individuelle.

Jusqu'à présent, l'ensemble des études qui cherchent à saisir comment se développent les compétences professionnelles aborde la question du point de vue de l'effectuation de l'action. Autrement dit, la compréhension des phénomènes de formation des compétences passe par une analyse des stratégies mobilisées dans et pour l'action à partir de laquelle s'opèrent ces activités productives. Par exemple, il s'agit de comprendre comment s'organisent les compétences produites dans le travail, comment créer un milieu et un environnement favorables à l'émergence des actions reconnues efficaces et comment concevoir des dispositifs de formation permettant l'acquisition et le développement des compétences professionnelles. 
Sans revenir sur l'histoire vive et polémique de cette notion $^{8}$, qui dépasserait le cadre de cet article, nous retiendrons la définition consensuelle que proposent Durat et Mohib (2008) de la compétence professionnelle comme "l'ensemble des ressources cognitives et affectives mobilisées et combinées par un individu pour agir de manière efficace et légitime dans une situation singulière ». Dans cette optique, on ne peut pas se contenter de considérer la compétence, inscrite du côté de l'action située et efficace, uniquement sous l'angle de l'action effectuée et réussie, c'est-à-dire de son résultat. Certes, la compétence se manifeste dans l'action (reconnaissance d'un savoir faire en acte) et fait corps avec l'action (routines, compétences incorporées) mais elle s'investit d'abord pour l'action conforme aux règles de l'art (mobilisation et ajustement des compétences pour agir dans une situation donnée). À vrai dire, la compétence repose sur un double mouvement opératoire. En même temps qu'elle se fonde sur la réalisation effective d'une action efficace et légitime, elle suppose un individu capable de s'engager dans l'agir professionnel. L'engagement est entendu ici au sens d' « acte de décision », tandis que l'agir professionnel peut être défini comme un « faire ordonné » qui renvoie à un ensemble d'actions potentielles conduites à la fois en fonction des intentions ou des valeurs du praticien, des exigences de la pratique et des contraintes relevant du contexte. Il apparaît ainsi que s'engager dans l'agir professionnel, c'est accepter de compter avec l'incertitude qui caractérise, pour reprendre Schön (1994), toute « pratique professionnelle ».

Dans la mesure où le développement des compétences implique un mouvement de propulsion de soi dans l'action, il importe d'en savoir plus sur ce qui favorise l'accomplissement d'un acte efficace et légitime. Notre angle d'approche est celui du pouvoir agir, et non pas du vouloir agir centré sur les facteurs motivationnels et qui est souvent privilégié dans le champ de la recherche pour aborder les questions d'engagement individuel, voire collectif. L'objectif de notre

\footnotetext{
${ }^{8}$ La notion de compétence a fait l'objet de nombreuses analyses dans des champs très variés tels que la linguistique, le droit, la gestion, la psychologie, la formation, la sociologie... Pour en savoir plus, $c f$. Aubret J., Gilbert P., Pigeyre F. (1993), Savoir et pouvoir : les compétences en questions, Paris, PUF ; Aubret J., Gilbert P. \& Pigeyre F. (2002), Management des compétences : réalisations-concepts-analyses, Paris, Dunod.
}

travail est d'explorer les conditions de développement des compétences professionnelles du point de vue de l'engagement dans l'agir, en nous focalisant à la fois sur le processus (approche identitaire) et les modalités de formation (approche instrumentale). Notre hypothèse est que pour s'engager dans l'agir professionnel, c'est-à-dire être capable d'entreprendre une action incertaine qui ne produit pas toujours les effets escomptés, l'individu doit se sentir autorisé par une « figure » qui fait autorité pour lui. Selon nous, l'acquisition des compétences professionnelles ne résulte pas d'une construction solipsiste. C'est pourquoi nous accordons une place importante au rôle des autres dans ce passage individuel de la prise de décision vers la réalisation effective. Le présent travail pourrait in fine contribuer à spécifier les modalités de construction des compétences et de leur genèse.

\section{UNE APPROCHE QUALITATIVE}

Notre travail repose exclusivement sur des données qualitatives dans la mesure où nous nous intéressons au sens que les individus accordent, selon le cas, à leurs pratiques ou à leurs manières d'agir.

\section{Trois terrains d'investigation}

Les terrains d'investigation renvoient à trois domaines différents de formation professionnelle de l'espace éducatif ( $c f$. tableau 1).

Le premier se rapporte à la formation d'étudiants inscrits dans la dernière année de leur cursus ( $5^{\mathrm{e}}$ année d'enseignement supérieur) et préparant leur diplôme d'ingénieur. La qualité des formations a toujours constitué une préoccupation majeure des écoles d'ingénieurs ; cependant, rares sont les travaux de nature éducationiste qui portent sur ce milieu où la question de la confrontation à la complexité de la réalité professionnelle demeure centrale.

Le deuxième terrain concerne la formation des professeurs stagiaires des lycées et collèges (PLC2) et des lycées professionnels (PLP2), organisée par 1'IUFM. L'acuité du débat sur la formation au métier d'enseignant, la crise identitaire que traverse, depuis quelques années, le milieu de l'enseignement, ou encore les 
Tableau 1

Caractéristiques générales des dispositifs de formation

\begin{tabular}{|c|c|c|}
\hline & Présentation et contexte & Objet d'étude \\
\hline $\begin{array}{l}\text { Instituts universitaires } \\
\text { de formation des maîtres } \\
\text { (IUFM) }\end{array}$ & $\begin{array}{l}\text { - Mis en place en } 1991 \text { (sous la tutelle } \\
\text { directe du ministre de l'Éducation nationale), } \\
\text { les IUFM ont changé de statut et sont devenus } \\
\text { des écoles internes de l'université à la suite } \\
\text { de la loi d'orientation et de programme pour } \\
\text { l'avenir de l'école de } 2005 \text {. } \\
\text { - Dispositifs de formation professionnelle: } \\
\text { initiale et continue } \\
\text { - Niveau de formation: Niveau I - Bac + } 5\end{array}$ & $\begin{array}{l}\text { - Année de professionnalisation et de } \\
\text { certification : } 2^{e} \text { année d'IUFM (formation } \\
\text { professionnelle initiale des enseignants). } \\
\text { - Acteurs institutionnels impliqués dans le } \\
\text { développement des compétences profes- } \\
\text { sionnelles des professeurs stagiaires: } \\
\text { directeur pédagogique, formateur/ } \\
\text { conseiller. }\end{array}$ \\
\hline École d'ingénieurs (EI) & $\begin{array}{l}\text { - Dispositifs de formation professionnelle : } \\
\text { initiale et continue } \\
\text { - Niveau de formation : Niveau I-Bac }+5 \text {. }\end{array}$ & $\begin{array}{l}\text { - Année de professionnalisation et de } \\
\text { certification : } 5^{e} \text { année d'enseignement } \\
\text { supérieur (formation professionnelle initiale } \\
\text { des ingénieurs). } \\
\text { - Acteurs institutionnels impliqués dans le } \\
\text { développement des compétences profes- } \\
\text { sionnelles des ingénieurs stagiaires: direc- } \\
\text { teur pédagogique, tuteur (enseignant ou } \\
\text { professionnel). }\end{array}$ \\
\hline $\begin{array}{l}\text { Maison des compagnons } \\
\text { du devoir (MCD) }\end{array}$ & $\begin{array}{l}\text { - Ces « maisons » sont des lieux } \\
\text { d'hébergement, de formation et d'échanges } \\
\text { qui accueillent des jeunes (18-25 ans) se } \\
\text { préparant à devenir Compagnon. } \\
\text { - Dispositifs de formation professionnelle : } \\
\text { initiale et continue } \\
\text { - Niveau de formation: Niveau V - BEP et/ } \\
\text { ou CAP en } 2 \text { ou } 3 \text { ans. }\end{array}$ & $\begin{array}{l}\text { - Année de professionnalisation et de } \\
\text { certification : CAP (certificat d'aptitude } \\
\text { professionnelle) « métiers de la construc- } \\
\text { tion » et «métiers de la mécanique ». } \\
\text { - Double projet pédagogique et moral. } \\
\text { - Acteurs institutionnels impliqués dans } \\
\text { le développement des compétences } \\
\text { professionnelles des apprentis: Prévôt } \\
\text { (Compagnon en fin de Tour de France, } \\
\text { gestionnaire de la MCDI. }\end{array}$ \\
\hline
\end{tabular}

Source : auteur.

récentes controverses dont font l'objet les IUFM avec la masterisation de la formation sont autant de raisons qui expliquent notre intérêt pour ce dispositif.

Le troisième terrain est relatif à la formation des « jeunes » dispensée dans les Maisons des compagnons du devoir (MCD); les modalités mises en œuvre chez les compagnons ${ }^{9}$ inspirent, depuis

\footnotetext{
${ }^{9}$ Pour résumer, le compagnonnage peut se définir comme le fruit d'une expérience professionnelle vécue corrélativement comme une expérience humaine où la qualification visée n'est pas seulement technique, mais aussi culturelle et morale. C'est autour de cet idéal, portant le compagnonnage comme un « esprit», que les Maisons du Compagnons du Devoir prônent encore aujourd'hui un certain nombre de principes tels que la vie en communauté, le sens de la responsabilité, le dépassement de soi, la fierté du métier ou encore la passion du travail. Le lecteur désireux d'en savoir plus sur ce sujet pourra se référer à Guédez (1994); Bayard J.-P. (1978); De Castéra B. (2008). Une bibliographie thématisée est également disponible sur Internet: cf. Dusantra, P. (1998). «Compagnonnage: que lire?». Renaissance traditionnelle,
}

quelques années, aussi bien les milieux de l'entreprise que ceux de l'enseignement professionnel ${ }^{10}$. Souvent associé à un groupement d'hommes dont l'origine se perd dans la nuit des temps, le compagnonnage en France a pendant longtemps été étudié sous le seul angle historique, en grande partie à cause de son rôle dans les mouvements ouvriers français. Pourtant, dès l'origine, la formation de l'individu représente le but ultime de cette organisation qui puise encore dans les traditions héritées du Moyen-Âge.

$\mathrm{n}^{\circ} 115 / 116$, pp. 299-305 in http://www.renaissancetraditionnelle.org/2010/11/sommaire-des-articles-du-tome-29-1998.html [dernière consultation le 26/04/2011].

${ }^{10}$ Aux États-Unis, par exemple, des enseignants-chercheurs travaillant dans des instituts technologiques multiplient les demandes d'information auprès des actuels compagnons français pour élaborer sur le même modèle la formation de techniciens supérieurs et de cadres-ingénieurs $C f$. (Mauriès, 2001). 
Pour chaque dispositif, à l'exception de la MCD pour laquelle notre investigation s'est limitée à une maison, nous avons retenu deux établissements ( $c f$. tableau 2) selon les critères suivants :

- Le premier critère renvoie à la diversité des situations de formation: la variété devait permettre de dégager des caractéristiques communes, mais aussi des éléments de différenciation des formes d'engagement dans l'agir professionnel. On y trouve en l'occurrence des dispositifs relatifs aussi bien aux métiers de l'humain qu'aux métiers techniques, des espaces de formation professionnelle initiale et continue, des contextes de formation inscrits dans une histoire récente ou au contraire plus ancienne, des terrains relevant du champ de l'enseignement supérieur et de celui de la formation professionnelle.

- Le deuxième critère concerne la présence d'une intention dominante de transformation et de professionnalisation des individus. En outre, tous les terrains choisis devaient présenter des formes d'activités et des démarches de formation articulées à la pratique et favorisant la construction des compétences professionnelles. Ont ainsi été retenus des dispositifs qui accordent une place privilégiée à l'agir dans la formation des futurs professionnels.

- Enfin, la présence des trois catégories d'acteurs impliqués dans le processus de construction individuelle des compétences professionnelles constitue le dernier élément de critérisation utilisé dans le cadre de notre stratégie d'échantillonnage. Sont concernés : les acteurs responsables de l'organisation et de la politique de formation des dispositifs dont dépendait l'accès à ces terrains (responsable pédagogique, directeur/trice des études...) ; les acteurs désignés par l'institution pour aider et soutenir les stagiaires dans leur confrontation avec le milieu de la pratique (tuteur/trice, formateur/trice, conseiller/ère...) ; les acteurs directement engagés dans les processus qui soutiennent leur engagement dans l'agir professionnel (stagiaire, jeune professionnel, néo-titulaire).

Le tableau 2 synthétise les caractéristiques générales pour chaque terrain visité au regard des critères ci-dessus ${ }^{11}$.

\footnotetext{
${ }^{11}$ Nous avons pris le parti de citer les terrains d'investigation en tenant compte de leur localisation régionale. Précisons que le choix de la région ne constitue pas un élément de critérisation.
}

Au fond, ce qui a guidé le choix des terrains, c'est l'importance accordée aux actions de développement des compétences professionnelles dans les dispositifs de formation. Dans notre approche, des éléments tels que le niveau de qualification, les modalités d'organisation propres à chaque terrain ou encore la nature des compétences spécifiques aux métiers de l'enseignement (IUFM), l'ingénierie (EI) et la construction ou la mécanique (MCD) ne constituent pas des variables déterminantes.

\section{Recueil et analyse des données}

Deux modes de recueil de données ont été privilégiés. Nous avons, dans un premier temps, recherché des informations relatives au fonctionnement général des dispositifs à partir de documents clés tels que les règlements pédagogiques et les plans de formation. Notre corpus comprend, au total, 12 documents d'une cinquantaine de pages en moyenne. Concrètement, l'analyse de ces textes nous a permis de rendre compte des logiques qui sous-tendent les actions de développement des compétences professionnelles et, notamment, des modalités pédagogiques instituées pour favoriser l'engagement des stagiaires dans l'agir professionnel.

Dans un deuxième temps, nous avons mené 22 entretiens semi-directifs ${ }^{12}$ répartis de la façon suivante : 8 avec d' " anciens stagiaires $»^{13}, 5$ avec les responsables pédagogiques (directeurs/trice des études), et 9 avec les référents pédagogiques (tuteur, accompagnateur, conseiller, formateur/trice...). Conscients du caractère prescriptif des documents pédagogiques, il

\footnotetext{
${ }^{12}$ Nos entretiens ont duré en moyenne 45 minutes, soit au total 16 heures d'enregistrements audio.

13 Les «anciens stagiaires" désignent ici des "professionnels » qui exercent une pratique, en moyenne depuis 4 ans, dans le domaine de compétences visées par le diplôme obtenu. Nous avons choisi de nous entretenir avec de jeunes praticien(ne)s plutôt qu'avec des personnes en cours de formation. Il nous semblait en effet important d'analyser l'expérience d'individus engagés en toute autonomie dans l'agir professionnel et pouvant de ce fait apporter un regard distancié et suffisamment critique sur leur formation initiale.
} 
Tableau 2

Caractéristiques générales des terrains d'investigation

\begin{tabular}{|c|c|c|}
\hline & Présentation du terrain & Critères de choix \\
\hline IUFM île de France & $\begin{array}{l}\text { - Effectif (étudiants + stagiaires) : } 5500 \\
\text { - Mise en place en } 2001 \text { d'un dispositif } \\
\text { d'accompagnement de l'entrée dans le métier à } \\
\text { destination des enseignants " néo-titulaires ». }\end{array}$ & $\begin{array}{l}\text { - Localisé dans « une des trois académies } \\
\text { qui accueille plus de la moitié de ceux } \\
\text { qui débutent » (1). } \\
\text { - Académie réputée pour ses zones } \\
\text { d'éducation prioritaire. } \\
\text { - Importance accordée par la direction } \\
\text { à la problématique de l'accompagnement } \\
\text { des enseignants. }\end{array}$ \\
\hline IUFM Rhône-Alpes & $\begin{array}{l}\text { - Développement, depuis 2002, } \\
\text { d'une politique de formation de professionnali- } \\
\text { sation des enseignants (ex. dispositif d'Accom- } \\
\text { pagnement à l'Entrée dans le Métier, séances } \\
\text { d'analyse des pratiques professionnelles, etc.). }\end{array}$ & $\begin{array}{l}\text { - Fait partie des trois établissements qui } \\
\text { ont ouvert à titre expérimental en } 1990 \\
\text { avant l'ouverture généralisée des IUFM } \\
\text { en } 1991 \text {. }\end{array}$ \\
\hline El Île de France & $\begin{array}{l}\text { - Établissement public à caractère scientifique et } \\
\text { culturel crée en } 1972 \text {. } \\
\text { - } 313 \text { enseignants-chercheurs pour } \\
3200 \text { étudiants, } 500 \text { ingénieurs diplômés } \\
\text { par an. } \\
\text { - Mise en place d'un enseignement à la carte: } \\
\text { ce sont les étudiants qui composent eux-mêmes } \\
\text { leur propre parcours de formation en choisissant } \\
\text { leurs enseignements. }\end{array}$ & $\begin{array}{l}\text { - Plusieurs fois classée } 2 \text { e école géné- } \\
\text { raliste dans le palmarès des meilleures } \\
\text { écoles d'ingénieurs après le bac (2). } \\
\text { - Double statut « dérogatoire »: cet } \\
\text { établissement fonctionne à la fois } \\
\text { comme une grande école et comme une } \\
\text { université. }\end{array}$ \\
\hline El Rhône-Alpes & $\begin{array}{l}\text { - Établissement public créé en } 1957 \text { sous tutelle } \\
\text { du ministère de l'Enseignement Supérieur } \\
\text { et de la Recherche. } \\
\text { - } 516 \text { professeurs permanents pour } \\
4106 \text { étudiants. } \\
\text { - } 900 \text { ingénieurs diplômés par an. } \\
\text { - admission à } 70 \% \text { au niveau Bac. }\end{array}$ & $\begin{array}{l}\text { - Plusieurs fois classée } 1^{\text {re }} \text { dans le } \\
\text { palmarès général au classement pédago- } \\
\text { gique et au classement des écoles les plus } \\
\text { profesionnalisantes (3). }\end{array}$ \\
\hline MCD Alsace et Lorraine & $\begin{array}{l}\text { - } 36 \text { professionnels au service des } \\
192 \text { « jeunes » (qui vivent sur place). } \\
\text { - } 400 \text { apprentis. } \\
\text { - } 50 \text { compagnons sédentaires (qui ont terminé } \\
\text { leur tour de France). } \\
\text { - Formation individuelle hors temps scolaire. }\end{array}$ & $\begin{array}{l}\text { - Importance accordée par la direction } \\
\text { aux questions de formation professionnelle } \\
\text { et d'accompagnement. }\end{array}$ \\
\hline
\end{tabular}

Sigles : IUFM : Institut universitaire de formation des maîtres. EI : École d'ingénieurs. MCD : Maison des compagnons du devoir.

(1) Eveleigh H.,Villatte N. (Éds) (2003), « Premiers pas dans l'enseignement ». Cahiers pédagogiques, 418.

(2) : Magazine Le Point (2004 - 2008)

(3) : Ibid.

Source : auteur.

nous a semblé important d'interroger aussi bien les responsables pédagogiques de chaque dispositif que les personnes censées, pour reprendre les termes de la plupart des textes institutionnels, «conseiller» et « soutenir» les futurs professionnels. Par ailleurs, notre échantillon est non représentatif mais expressif d'une diversité des regards, nécessaire à la mise à l'épreuve de l'hypothèse. Notre démarche visait en effet à rechercher le sens conféré au langage dans le contenu des expressions des locuteurs afin de mettre en lumière les systèmes de représentations véhiculés par leurs discours. En résumé, que disent-ils de ce qu'ils font et de comment ils font pour engager (anciens stagiaires) ou favoriser (acteurs institutionnels) des actions efficaces et légitimes? 
Pour traiter les données, nous avons utilisé une méthode d'analyse de contenu. Il s'agit d'identifier, d'une part, ce qui est mis en place par l'institution et, d'autre part, ce qui est mis en œuvre par l'individu pour développer des compétences professionnelles. Plus précisément, nous avons relevé de manière systématique l'ensemble des énoncés évoquant l'idée d'engagement dans l'agir professionnel. Nous avons ensuite procédé à un regroupement thématique qui a permis de faire ressortir les trois catégories suivantes : les déterminants de l'engagement dans l'agir professionnel, le rôle des tuteurs dans le développement du pouvoir «d'agir » des apprenants et le modèle de la formation aux compétences. Les caractéristiques individuelles des interviewés telles que l'âge, le sexe ou encore la catégorie socioprofessionnelle n'ont pas été prises en compte. En effet, elles ne constituent pas ici des variables explicatives de l'expression du discours.

\section{PAROLES D'ACTEURS}

\section{Les acteurs institutionnels}

Outre la diversité des domaines et des pratiques de formation, l'analyse des entretiens révèle une approche commune du développement des compétences, organisée autour de quatre modalités institutionnelles. Ces modalités se retrouvent aujourd'hui dans la quasi-totalité des discours qui accompagnent l'évolution des secteurs de notre vie (travail, emploi, santé éducation) : les mises en situation de responsabilité, l'injonction à la réflexivité, l'accompagnement et les incitations à échanger.

\section{Des mises en situation de responsabilité}

Le terme de « responsabilité » est un des mots les plus utilisés par les organisateurs des différentes formations professionnelles. Qu'il s'agisse des textes institutionnels ou des discours produits par les directeurs des études, tous insistent sur cette notion «aux contours théoriques plutôt flous » (Charbonneau \& Estèbe, 2001). Nous avons dénombré, au total, pas moins d'une quarantaine d'expressions associées à l'idée de « responsabilité », comme en témoignent les formulations suivantes: «Faut les responsabiliser, c'est tout (Prévôt, MCD) ; "La formation proposée en deuxième année s'appuie sur cinq principes : placer le/la stagiaire en position d'acteur responsable de sa formation » (Plans de formation 2003-2006, IUFM Rhône-Alpes) ou encore "Pour nous [l'objectif] c'est de les rendre responsables, qu'ils apprennent à prendre en charge des projets » (Directeur aux enseignements et à la pédagogie, EI Rhône-Alpes). D'un point de vue organisationnel, cette mise en situation de responsabilité des apprenants passe par l'organisation de stages, de projets, ou encore par l'engagement associatif, fortement encouragé dans les écoles d'ingénieurs.

\section{L'injonction à la réflexivité}

Les réflexions découlant de son expérience, l'explicitation de sa pratique professionnelle ou encore l'analyse distanciée de son action "située et singulière » constituent une autre dimension privilégiée dans la formation des futurs professionnels à la complexité de l'agir. Responsables institutionnels et référents pédagogiques sont unanimes quant au rôle de la réflexivité dans le développement des compétences professionnelles. Inciter les futurs professionnels à réfléchir et à analyser leurs actions constitue un autre moyen pour les apprenants de faire face aux difficultés qu'ils rencontrent in situ. Par exemple, à l'IUFM, « l'analyse des pratiques doit permettre aux jeunes enseignants de forger leur propre expérience en utilisant celle des plus expérimentés » (Plans de formation 2003-2006, IUFM Île de France). Des dispositifs pédagogiques sont également prévus pour développer la réflexivité des futurs ingénieurs et des compagnons sous la forme de bilans : «À la fin des deux semaines de formation, on fait un bilan, pour savoir où le jeune se positionne. Alors, ça peut être des bilans personnalisés, comme ça peut être des bilans en groupe » (Prévôt, MCD); " [de retour de stage] on les amène à se poser des questions sur la façon dont ils sont organisés, les problèmes qu'ils ont rencontrés. On les oblige à se poser des questions » (Directeur aux enseignements et à la pédagogie, EI Rhône-Alpes).

\section{L'accompagnement}

D'après la plupart des documents pédagogiques, ce sont principalement des fonctions d'aide et de conseil 
qui caractérisent le cœur du travail d'accompagnement. Ces résultats trouvent un écho dans les discours des référents pédagogiques eux-mêmes. Lorsque nous les avons interrogés sur leur rôle, tous ont d'abord mis en lumière leur fonction « d'orientation », « d'accompagnement», ou encore de « guidance ». Deux formes d'accompagnement se dégagent : le «modèle à suivre » ou le « suiveur ». Dans le premier cas, le stagiaire est accompagné par un référent qui va l'aider dans la maîtrise de situations complexes, notamment en le rassurant quant au bien-fondé de sa façon d'agir : «Nous, on les met en confiance. Après on les aide. Si jamais c'est pas réussi, c'est pas grave » (Compagnon plâtrier) ; " (...) le bénéfice que j'en retire, c'est de sentir que j'ai pu servir d'appui à quelqu'un qui va commencer à enseigner, qui s'est dit "tiens, ah oui, je pourrais faire comme ça parce qu'elle fait comme ça”... » (Tutrice, IUFM Île de France). Dans le second cas, le futur professionnel sera suivi par un accompagnateur davantage préoccupé par la transmission des savoirs formels «on leur transmet des notions très pointues (...) ce qu'on essaie de leur apporter, c'est un niveau théorique le plus haut possible pour qu'ils puissent vivre avec ce qu'ils ont appris le plus longtemps possible » (Enseignant-tuteur, EI Rhône-Alpes) ou encore le suivi administratif des apprenants que par l'engagement des stagiaires dans l'agir professionnel : «Le rôle consiste à récupérer des rapports (...) on a des fiches de suivi, des fiches avec lesquelles on communique avec les étudiants qui sont en stage (....) notre rôle, c'est vraiment de suivre. » (Enseignantsuiveur, EI Île de France)

\section{Les échanges}

Tous les responsables pédagogiques que nous avons interrogés ont déclaré que les échanges constituaient un élément primordial dans la formation des futurs professionnels. Tous nous expliquent qu'il faut inciter les stagiaires à échanger, les faire parler entre eux, voire avec d'autres personnes que celles qui sont prévues institutionnellement. C'est dans cette perspective que des rencontres informelles entre pairs sont favorisées : " L'intérêt entre pairs c'est qu'ils échangent entre eux et se rendent comptent, en première année en particulier, qu'ils ne sont pas seuls face à leurs difficultés et que ce n'est pas qu'a eux que ça arrive » (Directrice adjointe, IUFM Rhône-Alpes); "[dès leur retour de stage], on amène les étudiants à partager leurs expériences, à les faire parler de ce qu'ils ont vécu, comparer les différents systèmes de relation qu'ils ont pu avoir dans les entreprises des uns et des autres. » (Directeur de la formation, EI Île de France)

\section{Les anciens stagiaires}

Qu'il s'agisse d'activités techniques ou humaines, trois facteurs semblent favoriser l'engagement des anciens stagiaires dans l'agir professionnel: la confiance et mise en confiance en soi, l'expérience et le besoin de reconnaissance ou la quête de légitimité.

\section{La confiance et la mise en confiance en soi}

Le thème de la confiance, entendue ici comme un sentiment d'assurance quant à sa capacité à faire face aux différentes circonstances de la vie, est récurrent chez les professionnels interrogés. Les travaux de Bandura (2003), menés dans le champ de la psychologie sociocognitive, ont montré que croire que l'on peut modifier le cours de sa vie ou produire des effets sur son environnement augmente le niveau de pouvoir sur ses actions. Autrement dit, pour entreprendre une action, il faut d'abord croire que l'on est capable de l'accomplir. C'est dans ce sens que la quasi-totalité des anciens stagiaires se sont exprimés. Cependant, il convient d'opérer une distinction entre la « confiance en soi » de l'ordre de l'aptitude personnelle : « (...) je pense que c'est parce qu'on a confiance en soi qu'on peut les prendre (les décisions importantes) et qu'on arrive à se lancer [dans l'agir professionnel]. » (Professeure d'histoire-géographie, IUFM Île de France) et la «mise en confiance en soi » qui renvoie plutôt à une instance extérieure : "Quand on te met en confiance, ça va mieux (...) Moi, je suis un gars vraiment pas sûr de moi (...) après faut se lancer! C'est comme ça qu'on se lance! » (Plâtrier, $\mathrm{MCD})$. «A l'origine, je suis quelqu'un qui a assez confiance en moi mais ils [les formateurs de l'IUFM] nous ont vraiment donné confiance en nous mais donné confiance dans le sens ou ils nous donnaient les moyens de bien faire notre travail et... c'est ce qui nous met en confiance [pour agir] » (Professeure d'histoire-géographie, IUFM Île de France). 


\section{L'expérience}

La plupart des anciens stagiaires rencontrés reconnaissent l'expérience professionnelle comme un autre élément essentiel du passage à l'action. Selon eux, le recours à leurs acquis antérieurs leur permettrait de se sentir prêts à affronter des situations difficiles sans craindre les risques. Sur ce point, de nombreux travaux se sont déjà intéressés au rôle de l'expérience dans le développement cognitif des individus. Ils montrent que la répétition d'expériences de même nature génère des savoirs faire stabilisés. En se confrontant à des problèmes jugés analogues, le travailleur acquiert certains automatismes qui lui permettent d'adopter des solutions efficaces et d'anticiper par rapport à son action (Bonvalot, 1989). Ainsi, en réfléchissant sur leurs expériences antérieures, les anciens stagiaires ne sont pas seulement amenés à modifier leurs pratiques en vue d'acquérir de nouveaux "savoirs faire » et «comment faire" , mais également à développer leur " pouvoir d'agir ». " [Ce qui m'aide le mieux à prendre des risques] c'est l'expérience (...) je fais souvent une rétrospective en me disant ' $j$ 'ai déjà fait quelque chose qui ressemblait à ça" puis j'essaie de me souvenir comment je l'avais fait, quels moyens j'avais pris pour le faire et puis je le fais! » (Mécanicien, MCD).

\section{Le besoin de reconnaissance ou la quête de légitimité}

Tous les anciens stagiaires rencontrés ont exprimé, de façon unanime, la quête de légitimité comme un élément déterminant de leur engagement dans l'agir incertain. Si « le sujet individuel se considère souvent comme la cause de sa propre action $»$ (Barbier, 2000), les acteurs interrogés reconnaissent leur besoin de se référer à autrui pour agir lorsqu'ils sont confrontés à des situations complexes. Au-delà d'un simple retour réflexif ou d'une rencontre opportune favorisant la mise en discours de leurs actions, il semble que ces échanges apportent avant tout une forme de soutien, voire de réconfort.

Nous avons identifié trois catégories de référents auprès desquels les anciens stagiaires «s'autorisent » pour s'engager dans l'agir professionnel : les collègues de travail, les pairs (membres du groupe de formation de l'institution d'origine) et moins largement la figure d'autorité (un ancien stagiaire sur deux a déclaré s'être référé à l'un de ses formateurs ou patrons pour développer son pouvoir d'agir). Dans tous les cas, ce mode de désignation relève d'un processus individuel spontané et repose sur des critères purement subjectifs.

Deux critères ont ainsi pu être mis en évidence dans notre étude : la dimension affective de la relation à l'autre et le partage d'une vision du monde. Les anciens stagiaires interrogés recherchent une forme d'autorisation, soit auprès des personnes avec qui ils s'entendent bien : «Quand j'ai un problème, j'appelle quelqu'un que je connais, qui a fini son tour, qui est sédentaire - on appelle ça un Compagnon sédentaire - avec qui je m'entends bien » (Plâtrier, MCD) soit auprès de celles qui partagent les mêmes conceptions et les mêmes façons de faire : "L'affinité c'est évident que ça joue, il y a des profs qui ont les mêmes méthodes que moi et je sais qu'ils pourront m'apporter des conseils intéressants » (Professeure d'histoire-géographie, IUFM Île de France). Ces personnes peuvent bien sûr être plus expérimentées, mais ce critère ne constitue pas une condition nécessaire. $\mathrm{La}$ légitimité des référents ne s'appuie pas forcément sur un indicateur de reconnaissance sociale (compétence, niveau d'expérience, statut social, etc.). «ça peut-être n'importe qui dans le groupe (...) même des fois des plus jeunes » (Mécanicien, MCD).

\section{Regards croisés d'acteurs}

Il est intéressant de croiser les regards des acteurs institutionnels et des anciens stagiaires, comme nous le proposons dans le tableau $\mathbf{3}$, qui indique comment nous regroupons les résultats présentés précédemment. Ainsi, les déterminants de l'engagement dans l'agir professionnel tiennent à deux éléments :

- l'autonomie encouragée par les acteurs institutionnels et revendiquée par les anciens stagiaires. On retrouve là le modèle dominant de l'individu réflexif et rationnel qui est mis en avant à tous les niveaux de la vie sociale.

- l'aide à la gestion de l'incertitude de l'action à travers des interactions sociales plus ou moins organisées du côté institutionnel, sollicitées du côté des anciens stagiaires. 
Tableau 3

Déterminants de l'engagement dans l'agir : regards croisés

\begin{tabular}{|l|l|l|}
\cline { 2 - 3 } \multicolumn{1}{c|}{} & \multicolumn{1}{c|}{ Acteurs institutionnels } & \multicolumn{1}{c|}{ Anciens stagiaires } \\
\hline \multirow{2}{*}{ Autonomie } & Mise en situation de responsabilité & Confiance et mise en confiance \\
\cline { 2 - 3 } & Réflexivité & Expérience \\
\hline $\begin{array}{l}\text { Aide à la gestion de l'incertitude } \\
\text { de l'action }\end{array}$ & $\begin{array}{l}\text { Accompagnement (tuteurs, suiveurs, } \\
\text { conseillers...) }\end{array}$ & $\begin{array}{l}\text { Besoin de reconnaissance ou quête } \\
\text { de légitimité }\end{array}$ \\
\cline { 2 - 2 } & $\begin{array}{l}\text { Échanges non formalisés (pairs, } \\
\text { professionnels...) }\end{array}$ & \\
\hline
\end{tabular}

Source : auteur.

Pour revenir à notre hypothèse, ce travail montre que les formes de légitimation dans lesquelles s'ancre la décision d'agir renvoient davantage à une quête de socialisation et de reconnaissance par les autres qu'à une personne référente pour l'individu. Autrement dit, pour s'engager dans l'agir professionnel, l'individu a besoin de se sentir autorisé par un groupe auquel il se réfère plus qu'à une figure qui fait autorité pour lui.

Au-delà de l'idée que les jeunes professionnels ont besoin d'être rassurés et de sentir qu'ils ne sont pas les seuls à vivre des difficultés, nous retiendrons que les anciens stagiaires que nous avons rencontrés s'inscrivent tous dans une quête de légitimité. Ce résultat est intéressant pour deux raisons :

- il permet de relativiser l'image du nouvel individu d'aujourd'hui valorisé, «souple, mobile, autonome, indépendant, [qui] trouve par lui-même ses repères dans l'existence et se réalise par son action personnelle » (Ehrenberg, 1991);

- la référence au groupe illustre bien ce passage d'une société des pères à une société des aînés qu'évoque Boutinet dans un entretien récent sur les problématiques que soulève le recours à l'accompagnement aujourd'hui (Bourdoncle et Gonnin-Bolo, 2009).

Pourtant, les institutions persistent (à l'exception de la $\mathrm{MCD}$ ) à accorder une place privilégiée à l'accompagnateur, au détriment du groupe informel dont le rôle est unanimement reconnu dans le développement du pouvoir d'agir des stagiaires : "Pour mener à bien [son] travail, chaque stagiaire est aidé par un conseiller pédagogique» (Livret du conseiller pédagogique, IUFM Île de France) ; "On attache beaucoup d'importance à l'aspect "suivi" avec différents moyens, avec visite (...) avec suivi électro- nique aussi » (Directeur aux enseignements et à la pédagogie, EI Rhône-Alpes). Que faut-il lire dans ce maintien d'une figure fragilisée dans une société en déficit d'autorité ? La simple reconnaissance d'une autorité fondée sur la compétence de son détenteur? Le renforcement d'une tension entre l'aide à la gestion de l'incertitude de l'action et l'injonction à l'autonomie ? Certains référents pédagogiques considèrent, par exemple, qu'ils n'ont aucun impact sur la capacité à s'engager de leurs stagiaires : «Non, moi je n'interviens jamais pour aider le stagiaire [à s'engager dans l'agir professionnel], j'interviens pour donner conseil. » (Enseignant-suiveur, EI Île de France)

Dans tous les cas, s'il faut accompagner les stagiaires, il faut surtout les responsabiliser, nous confient les personnes chargées de l'organisation de la formation. On ne peut s'empêcher ici de faire un rapprochement avec les transformations actuelles liées à la généralisation des valeurs de l'autonomie à tous les niveaux de la société individualiste (école, santé, travail...). D'une certaine manière, cette injonction à la responsabilité semble contradictoire si l'on considère que l'autonomie est à la fois une condition et une visée de la formation professionnelle.

Notre travail révèle que cette responsabilisation des acteurs passe par l'organisation de stages, le développement de projets collectifs ou individuels qui supposent déjà une autonomisation des individus euxmêmes, comme en témoignent les propos suivants : «Les gens (les étudiants) doivent être autonomes, dès qu'ils rentrent ici [en école d'ingénieurs] il faut qu'ils soient autonomes... une personne non autonome n'a quasiment aucune chance d'arriver en cinquième année (...) c'est normal d'être autonome, 
c'est une condition nécessaire. ॥ (Enseignant-tuteur, EI Rhône-Alpes)

À cet égard, les anciens stagiaires peinaient à formuler leur besoin de reconnaissance pour développer leur pouvoir d'agir. À les écouter, il semble plus facile de parler du sens que l'on donne à ce qu'on fait, de dire comment on s'y prend pour réussir, mais révéler ce qui permet «d'oser faire » laisse perplexe tous ceux que nous avons interrogés. Pour celui qui « fait» et qui « agit », il apparaît difficile d'expliquer comment il s'autorise à se lancer dans telle direction plutôt que dans une autre ou encore de décrire ce qui lui permet de dépasser ses craintes et ses hésitations liées au caractère imprévisible de l'action. Sans doute qu'une telle révélation peut être considérée comme le signe d'une faiblesse à une époque où il faut sans cesse prouver son efficacité et sa capacité à dominer toute situation nouvelle. Un des ingénieurs diplômé de l'EI Rhônes Alpes a répondu que le fait de se référer à ses collègues «n'avait rien à voir avec le besoin d'être rassuré » mais que c'était un moyen "de combler les $10 \%$ de lacunes » qu'un jeune professionnel peut avoir.

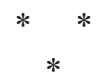

Au terme de cette analyse, soulignons que la question de l'engagement dans l'agir professionnel a suscité un grand intérêt de la part des acteurs institutionnels interrogés. En effet, elle constitue pour eux une préoccupation majeure, notamment pour les organisateurs des formations. Toutefois, les interviewés n'ont pas répondu aisément à nos questions. Il a fallu sans cesse reprendre certaines de nos questions et les reformuler tout en les adaptant à l'interlocuteur. Là où les mots « problème », « responsabilité », « travail » prenaient tout leurs sens, ceux d' « engagement» ou encore d' « agir professionnel» sont apparus déroutants. La confusion entre « pouvoir agir » et « vouloir agir », l'absence d'un référentiel langagier commun concernant l'agir professionnel, déjà signalé par Barbier (2000), sont d'autres éléments qui témoignent de la complexité même de l'objet étudié.

S'ajoutent à ces difficultés des limites méthodologiques et théoriques que nous souhaitons préciser :

- Limites méthodologiques car malgré la richesse des informations obtenues, liée à la souplesse même de la méthode, nous regrettons l'absence d'observations de mise en situation réelle des acteurs qui auraient permis d'en dire plus sur les conditions de l'engagement dans l'agir professionnel. Nous avons également fait le choix d'une approche analytique en nous centrant sur le rôle des acteurs, sans tenir compte des données personnelles des individus ni même de la spécificité des terrains et des métiers préparés. De telles données permettraient sans doute d'analyser plus finement les modalités de cette quête de légitimité que nous avons mise en évidence.

- Limites théoriques également puisque le cadre que nous avons retenu pour appréhender le modèle de développement des compétences professionnelles ignore les dimensions du savoir et du vouloir agir. L'absence de liens entre cognition, affect, objectif et action laisse à penser que ce modèle d'analyse est réduit, voire trop réduit. En même temps, pour reprendre Astolfi (2003), n'est-ce pas «le propre de toute activité modélisante que de simplifier l'objet pour mieux l'analyser, dès lors qu'on ne prend pas le modèle pour la réalité »?

- L'intérêt principal de cette recherche tient d'abord à son objet d'étude. La question du développement des compétences professionnelles sous l'angle du pouvoir d'agir est peu explorée ; la plupart des études restent centrées sur les capacités disciplinaires ou sur ce que Le Boterf (1994) et Perrenoud (2000) appellent les « ressources cognitives ».

- Une autre originalité de ce travail est de confronter des modèles de formation qui relèvent de champs professionnels différents (enseignants, ingénieurs, compagnons) et habituellement analysés de manière indépendante. Au fond, ce qui est spécifique à chacun de ces espaces de formation, c'est l'agir professionnel et non pas l'engagement dans l'agir. Dans une culture de l'acteur, nous faisons l'hypothèse que l'individu a besoin de se sentir autorisé par un groupe auquel il se réfère plus qu'à une figure qui fait autorité pour lui. Dès lors, n'est-ce pas le modèle dominant du développement des compétences qui soutiendrait la perte de légitimité de l'accompagnateur institutionnel au profit d'un accompagnement collectif par le groupe ? Aujourd'hui, les bénéfices de ces interactions sociales sont reconnus s'agissant de l'apprentissage. Qu'en est-il de leurs apports sur le développement de cette capacité à s'engager dans l'agir ? C'est une remise en 
perspective de la finalité de la formation professionnelle, « on n'apprend pas seul, on ne se forme pas seul mais on agit par soi-même », que cette recherche nous invite à réaliser.

\section{Bibliographie}

Astier P. (2009), «Travail et formation » in Barbier J.-M., Bourgeois E., Chapelle G., Ruanao-Borbalan J.-C. (Éds), Encyclopédie de la formation, Paris, PUF, pp. 733-748.

Astolfi P. (2003), Éducation et formation : nouvelles questions, nouveaux métiers, Paris, ESF.

Baillauquès S. (Éd.) (2001), La personnalisation d'une formation professionnelle. Le cas des professeurs des écoles, Paris, INRP.

Bandura A. (2003), Auto-efficacité : le sentiment d'efficacité personnelle, Paris, De Boeck.

Barbier J.-M. (2000), L'analyse de la singularité de l'action, Paris, PUF.

Barbier J.-M. (2001), « La formation des adultes : crise et recomposition » in CNA-Centre de recherche sur la formation (Éd.), Questions de recherches en éducation - 2 - Action et identité, Paris, INRP, pp. 11-25.

Barbier J.-M. (2009), « Le champ de la formation des adultes », in Barbier J.-M., Bourgeois E., Chapelle G., Ruanao-Borbalan J.-C. (Éds), Encyclopédie de la formation Paris, PUF, pp. 1-28.

Barbier J.-M., Berton F., Boru J.-J. (1996), Situations de travail et formation, Paris, L'Harmattan.

Bayard J.-P. (1978), Le compagnonnage en France, Paris, Payot.

Bonvalot G. (1989), «L'entreprise, espace de formation expérientielle», Éducation permanente (100/101), pp. 51-160.
Bourdoncle R., Gonnin-Bolo A. (2009), «Entretien avec Jean-Pierre Boutinet », Recherche et Formation, 62, pp. 109-124.

Carré P. (2005), L'apprenance: vers un nouveau rapport au savoir, Paris, Dunod.

Charbonneau J., Estèbe P. (2001), « Entre l'engagement et l'obligation: l'appel à la responsabilité à l'ordre du jour », Liens social et politiques, 46. Repéré à http://www.erudit.org/revue/lsp/2001/v/ n46/000319ar.pdf

De Castéra B. (2008), Le compagnonnage : culture ouvrière, Paris, PUF.

Dietrich A. (1999), «La dynamique des compétences, point aveugle des techniques managériales », Formation Emploi, 67, pp. 9-23.

Dubar C. (1996), « La sociologie du travail face à la qualification et à la compétence », Sociologie $d u$ travail, 2, pp. 179-193.

Dugué E. (1994), « La gestion des compétences : les savoirs dévalués, le pouvoir occulté », Sociologie $d u$ travail, (3), pp. 273-292.

Dugué E. (1999), «La logique de la compétence: le retour du passé », Éducation permanente 140, pp. 7-18.

Durand M. (2009), « Analyse du travail dans une visée de formation : cadres théoriques, méthodes et conceptions » in Barbier J.-M., Bourgeois E., Chapelle G., Ruanao-Borbalan J.-C. (Éds), Encyclopédie de la formation, Paris, PUF, pp. 827-856. 
Durand M., Sève C., Saury J. \& Theureau J. (2002), «La construction de connaissances chez des sportifs de haut niveau lors d'une interaction compétitive », Le travail humain, 2(65), pp. 159-190.

Durat L., Mohib N. (2008), « Le développement des compétences au regard de l'engagement dans l'agir professionnel », Questions Vives, 10, pp. 25-41.

Ehrenberg A. (1991). Le Culte de la performance, Paris, Calmann-Lévy.

Guédez A. (1994), « Compagnonnage et apprentissage », Paris, PUF.

Le Boterf G. (1994), « De la compétence : essai sur un attracteur étrange », Paris, Éditions d'organisation.

Lichtenberger Y. (1999), « Compétence, organisation du travail et confrontation sociale », Formation Emploi, 67, pp. 93-109.

Livian Y.-F., Terrenoire J. (1995), « Les entreprises face aux exigences de la gestion des compétences », Personnel, 361, pp. 59-61.

Marcel J.-F., Orly P., Rothier-Bautzer E., Sonntag M. (2002), «Les pratiques comme objet d'analyse», Revue française de pédagogie, 138, pp. 135-170.

Mauriès F. (2001), «Dispositifs de “compagnonnage" pour développer les compétences techniques en situation de travail », Actualité de la formation permanente, 170 , pp. 50-54.

Oiry E. \& Iribarne (d’) A. (2001), « La notion de compétence : continuité et changements par rapport à la notion de qualification ", Sociologie du travail. 43(1), pp. 49-66.

Olry P. (2003), « Organisation du soin et développement des compétences : contribution à la professionnalisation en masso-kinésithérapie », Les sciences de l'éducation - Pour l'ère nouvelle, 36(2), pp. 91-105.

Paradeise C., Lichtenberger Y. (2001), « Compétence, compétences», Sociologie du travail, 43(1), pp. 33-48.
Pastré P. (1999), « Travail et compétences : un point de vue de didacticien », Formation Emploi 67, pp. 109-125.

Pelpel P. (2001), Apprendre et faire. Vers une épistémologie de la pratique?, Paris, L'Harmattan.

Perrenoud P. (2004), Dix nouvelles compétences pour enseigner : invitation au voyage, Paris, ESF.

Perrenoud P. (2000), «L'approche par compétences : une réponse à l'échec scolaire? » AQPC Réussir au collégial. Communication présentée aux Actes du Colloque de l'association de pédagogie collégiale, Montréal, Canada. Repéré à http://www. unige.ch/fapse/SSE/teachers/perrenoud/php_main/ php_2000/2000_22.html

Perrenoud P., Altet M., Charlier E., Paquay Y.-L. (1998), «Fécondes incertitudes ou comment former des enseignants avant d'avoir toutes les réponses ? » in Paquay L., Altet M., Charlier E., Perrenoud P. (Éds), Former des enseignants professionnels: quelles stratégies?, Quelles compétences?, Bruxelles, De Boeck, pp. 239-253.

Rabardel P., Six B. (1995), « Outiller les acteurs de la formation pour le développement des compétences au travail », Éducation permanente, 123, pp. 33-43.

Raucent B., Vander Borght C. (Éds) (2006), Etre enseignant : Magister? Metteur en scène? Bruxelles, De Boeck.

Schön R. (1994), Le praticien réflexif : à la recherche du savoir caché dans l'agir professionnel, Montréal, Éditions Logiques.

Wittorski R. (1998), « De la fabrication des compétences », Éducation permanente, 2(135), pp. 57-69.

Wittorski R. (2009), «A propos de la professionnalisation » in Barbier J.-M., Bourgeois E., Chapelle G., Ruanao-Borbalan J.-C. (Éds), Encyclopédie de la formation, pp. 781-792, Paris, PUF. 


\title{
Résumé
}

\section{Développer des compétences ou comment s'engager dans l'agir professionnel}

\author{
Najoua Mohib
}

Issu d'une recherche en sciences de l'éducation, cet article analyse le développement des compétences professionnelles dans des dispositifs de formation visant à professionnaliser des stagiaires. Trois types d'espaces ont été investigués: la Maison des compagnons du devoir, les IUFM (Instituts universitaires de formation des maîtres) et les écoles d'ingénieurs. Plutôt que d'aborder le développement des compétences du point de vue de l'effectuation de l'action, celui-ci est exploré sous l'angle du passage à l'action. Dans la mesure où la compétence suppose l'engagement d'un individu dans l'agir, il importe d'explorer les modalités de formation et les processus qui soutiennent cet engagement. Alors que les discours institutionnels insistent sur la capacité à agir par soi-même, les anciens stagiaires (jeunes professionnels) expriment leur besoin de reconnaissance par les autres plutôt que par une figure d'autorité.

\section{Mots clés}

Compétence, savoir professionnel, stagiaire, processus d'apprentissage

Journal of Economic Literature : J 24 